\title{
Kyste nasopalatin associé à une dent surnuméraire incluse : à propos d'un cas
}

\author{
Nasopalatine duct cyst associated with a supernumerary impacted teeth: \\ a case report
}

BASSIMA CHAMI*, WAFAE EL WADY*

\begin{abstract}
RÉSUMÉ
Dans la classification de l'OMS de 1992, le kyste naso-palatin fait partie des kystes malformatifs non odontogènes des maxillaires. II représente la variété la plus fréquente de ces kystes. Son étiopathogénie est incertaine. Son diagnostic positif repose sur des signes cliniques habituellement discrets et sur des signes radiographiques le plus souvent révélateurs du kyste qui se traduit par une image radiotransparente en forme de cœur de carte à jouer, et parfois par une divergence des racines des incisives centrales. Le diagnostic est confirmé par l'examen histopathologique. La difficulté soulevée par le cas rapporté est celle du diagnostic différentiel à partir des seules données cliniques et radiographiques, soulignant ainsi l'intérêt de l'examen histopathologique. (Med Buccale Chir Buccale 2005; 11: 31-37).
\end{abstract}

mots clés : Kyste nasopalatin, dent surnuméraire, kystes non odontogènes

médecine buccale chirurgie buccale

VOL. $11, \mathrm{~N}^{\circ} 1$ 2005

page 31

\section{SUMMARY}

The nasopalatine duct cyst (NPDC) is a nonodongenic cyst according to the world health classification 1992. It represents the most common maxillary nonodontogenic cyst. It's accuracy accounts for 0,08 to 1,5\%. Etiopathogenesis is still widly debated. Since the clinical symptoms are often relatively discrete, radiography apperence as a powerfull tool in diagnosis. Indeed, NPDC usually appear as an around, ovoid or heart shaped radiolucency located between the roots of central incisors. Nevertheless, the indispensable histopathological analysis must be conducted to confirm the diagnosis. The aim of this article was to precise the difficulty of differentiating the nasopalatine duct cyst from some of odontogenic cysts (calcifying cyst, dentigerous cyst) if diagnosis is based on clinical and radiographic symptoms alone showing the importance of the histopathological analysis. (Med Buccale Chir Buccale 2005; 11: 31-37).

key words : Nasopalatine duct cyst, supernumerary teeth, nonodontogenic cyst 
médecine buccale chirurgie buccale

VOL. $11, \mathrm{~N}^{\circ} 1$ 2005

page 32
Les kystes non odontogènes, improprement appelés kystes fissuraires (1972), sont constitués par une cavité contenant une collection liquidienne, bordée par un épithélium non odontogène. Cet épithélium provient de cellules épithéliales séquestrées lors du développement des bourgeons maxillofaciaux [1]

Dans la récente classification de l'OMS (1992), le terme de kyste malformatif non odontogène a remplacé celui de kyste fissuraire [2]. Dans ce groupe, sont décrits les kystes nasopalatins (KNP) et les kystes nasolabiaux.. Les autres kystes (médiopalatins, médio-mandibulaires et globulomaxillaires), inclus jusqu'ici dans le groupe des kystes fissuraires, ont été écartés dans la nouvelle classification [2].

Les kystes non odontogènes sont rares puisqu'ils ne représentent que $5 \%$ des kystes maxillaires [1]. Leur étiologie explique leur localisation particulière [3]. Cependant, comme dans le cas de l'observation rapportée, il peut y avoir des difficultés lors du diagnostic différentiel avec les kystes odontogènes. C'est dire l'importance de l'examen histopathologique pour le diagnostic définitif, car les renseignements cliniques et radiologiques sont insuffisants pour un diagnostic précis [2].

\section{OBSERVATION}

Un homme, âgé de 19 ans, vient en consultation pour une tuméfaction labiale supérieure entraînant une gène esthétique et fonctionnelle. Le patient est en bon état général, sans antécédent particulier. II signale plusieurs épisodes inflammatoires en rapport avec cette tuméfaction, qui ont régressé rapidement après la prise d'antibiotiques. L'examen clinique exobuccal montre une discrète tuméfaction labiale supérieure. L'examen endobuccal révèle une tuméfaction comblant le vestibule en regard des incisives supérieures, de consistance ferme, légèrement dépressible en son centre et recouverte d'une muqueuse d'aspect normal (Fig. 1). Les dents en rapport avec la tuméfaction $(11,12,21$, et 22) sont saines et présentent une mobilité de degré 1 . Les tests de vitalité montrent une hypersensibilité de 11, 14, 22 et 23, une absence de sensibilité de 12 et 21 et une légère sensibilité de la 13. Le reste de l'examen endobuccal est sans particularité.
Une radiographie rétroalvéolaire et un orthopantomogramme montrent une image radiotransparente, à contour légèrement festonné, en forme de cœur de carte à jouer, siégeant entre les racines divergentes des deux incisives centrales supérieures, avec la présence en son centre d'une masse radioopaque ressemblant à une dent surnuméraire incluse, en position inversée (Fig. 2). La radiographie occlusale montre une lésion très étendue, allant de 14 à 21, qui semble respecter les fosses nasales (Fig. 3).

Devant ces éléments cliniques et radiologiques, plusieurs hypothèses diagnostiques sont évoquées :

- un kyste nasopalantin en raison de la forme et du siège médian de l'image radiographique,

- un kyste péricoronaire développé à partir d'une dent surnuméraire incluse,

- un kyste odontogène calcifié devant cette lésion constituée par une masse radio-opaque au centre d'une image radiotransparente.

Quel que soit le diagnostic, le traitement de cette lésion est chirurgical. II consiste en l'énucléation totale du kyste avec examen histopathologique de la pièce opératoire. Les différents temps opératoires sont identiques à ceux réalisés pour les autres kystes (Fig. 4, 5, 6).

Après fixation dans du formol tamponné, la pièce opératoire (Fig. 7), qui mesure $5 \mathrm{~cm}$ dans son grand axe, est examinée au microscope. La paroi kystique est constituée d'un revêtement à la fois malpighien et cylindrique pseudo-stratifié cilié de type respiratoire ; cet aspect est caractéristique d'un kyste nasopalatin (Fig. 8, 9). Dans la paroi conjonctive, on peut observer une réaction granulomateuse autour de cristaux de cholestérol (Fig.10).

\section{COMMENTAIRES}

Selon la classification de l'OMS (1992) [4], le kyste du canal nasopalatin ou kyste du canal incisif, plus communément appelé kyste nasopalatin (KNP), fait partie des kystes non odontogènes. II représente la variété la plus fréquente de ces kystes [5]. Son incidence varie entre 0,08 et $1,5 \%$ selon les auteurs [6,7]. II a été décrit pour la première fois par Meyer en 1914 qui l'a assimilé à un sinus nasal surnuméraire ${ }^{[4,8]}$. Les termes de kyste médian palatin ou alvéolaire et de kyste de la papille palatine sont synonymes du KNP $[4,6,7]$. 


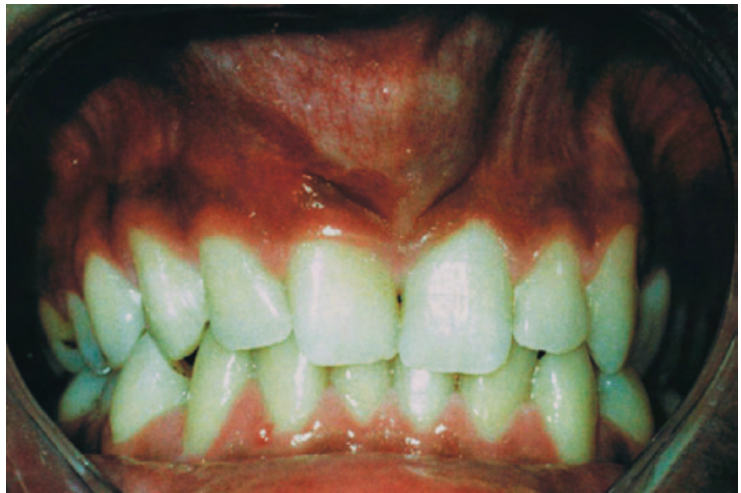

Figure 1: Vue endobuccale montrant une tuméfaction en regard des incisives supérieures

Intraoral view: a vestibular tumefaction is seen in the upper incisive region

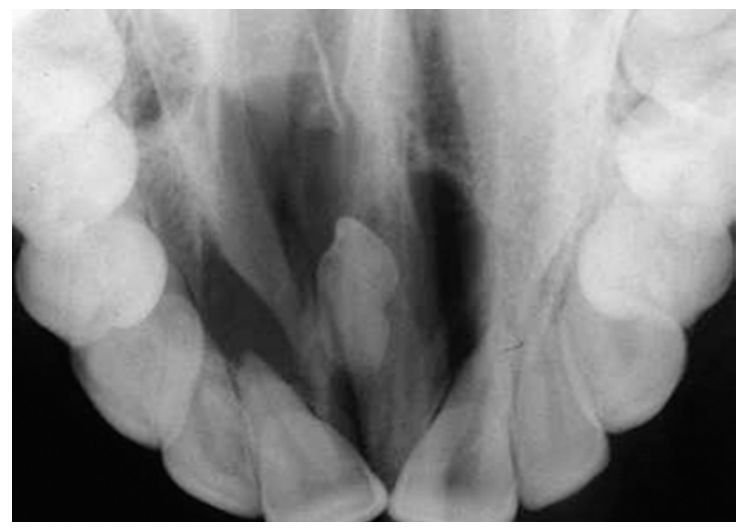

Figure 3: Radiographie occlusale montrant un aspect identique

Maxillary occlusal showing a same radiographic aspect

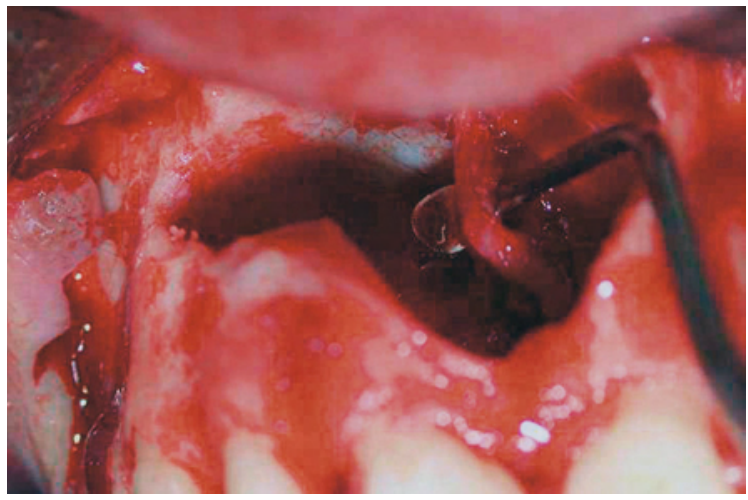

Figure 5: Vue endobuccale montrant le nerf nasopalatin

Intraoral view showing the nasopalatine nerve

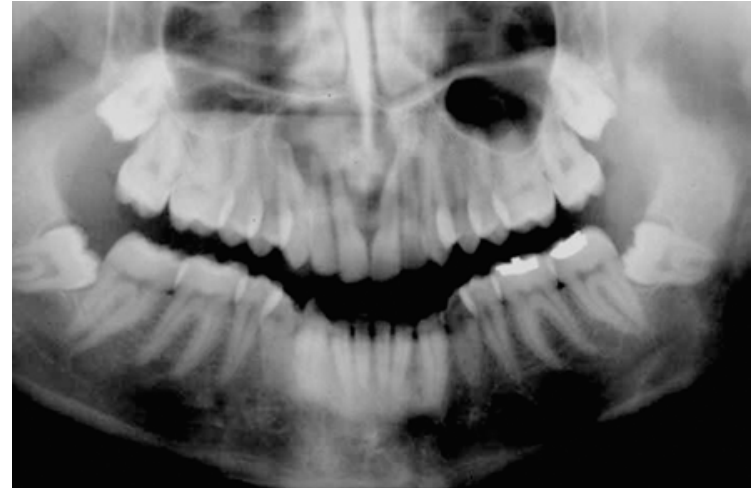

Figure 2: L'orthopantomogramme donnant une vue globale de la lésion

Orthopantomography showing the lesion area

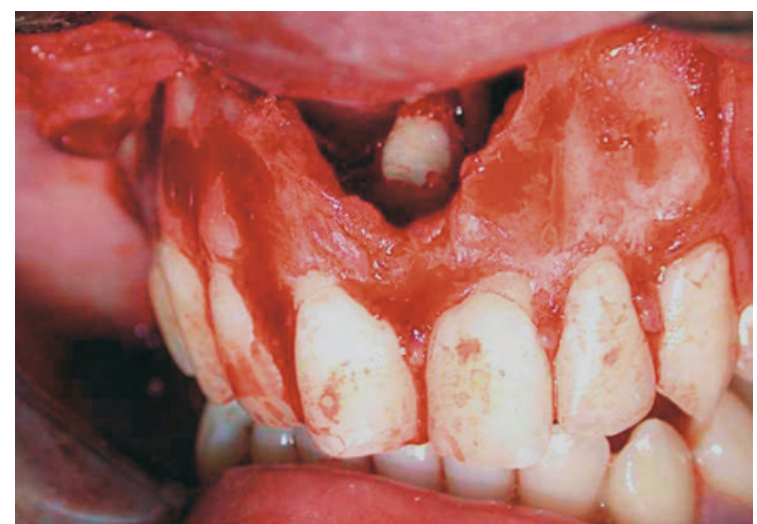

médecine

buccale

chirurgie

buccale

VOL. $11, N^{\circ} 1$

2005

page 33
Figure 4: Vue endobuccale montrant la position de la dent surnuméraire dans la cavité kystique Intraoral view: the supernumerary teeth settled in the middle of the cyst cavity

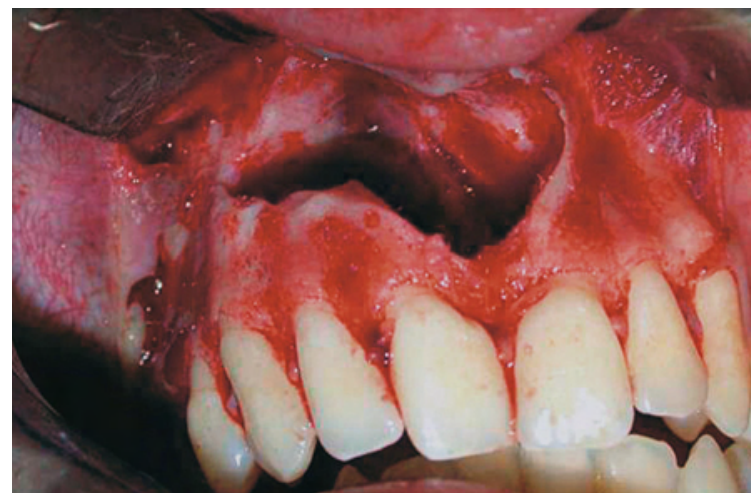

Figure 6: Vue endobuccale après énucléation totale du kyste

Intraoral view after total enucleation 


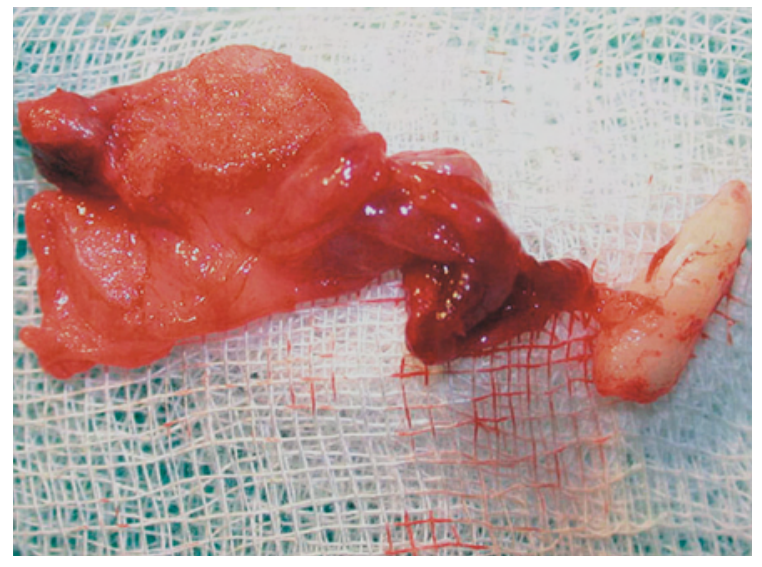

Figure 7: La pièce opératoire : kyste associé à une dent surnuméraire

Operative view: the cyst associated with the supernumerary teeth

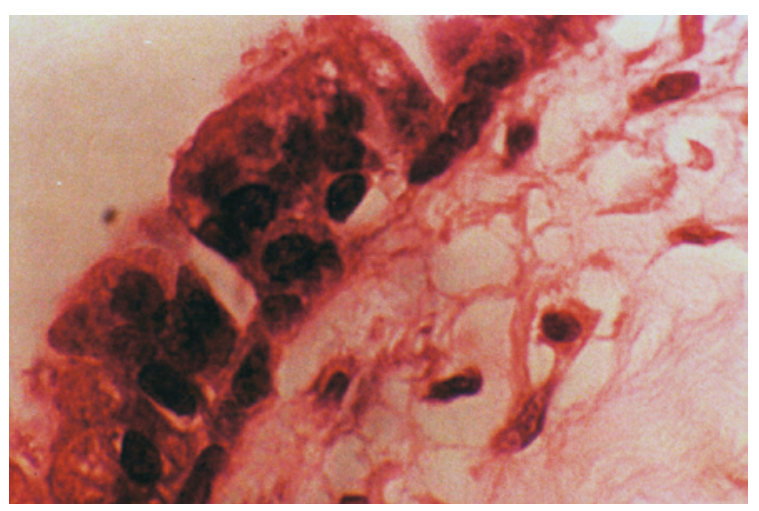

Figure 9 : Paroi du kyste avec un revêtement cylindrique pseudostratifié cilié de type respiratoire (coloration $\mathrm{HE}$ ) (Immersion, x 100)

The cyst wall lined by pseudostratified ciliated epithelium (HE $\times$ 100)

Ce kyste serait plus fréquent chez les hommes : le sexe ratio varie entre $1.7 / 1$ et $3 / 1$ [6, 8, 9]. Le KNP peut survenir à n'importe quel âge, même chez un fœtus [6, 8]. Cependant les tranches d'âge les plus concernées sont les $4 \mathrm{e}$, 5eet 6 e décennies $[4,5,6,7]$. Certains auteurs signalent une plus grande fréquence du KNP chez les patients afrocarraibiens pendant la cinquantaine alors que, pour les caucasiens, ce serait plutôt la $6^{\mathrm{e}}$ décennie [8]. La survenue du KNP chez les enfants ou chez les adultes jeunes n'est pas exceptionnelle puisqu'on retrouve dans la littérature plusieurs cas de KNP chez des patients âgés de huit et neuf

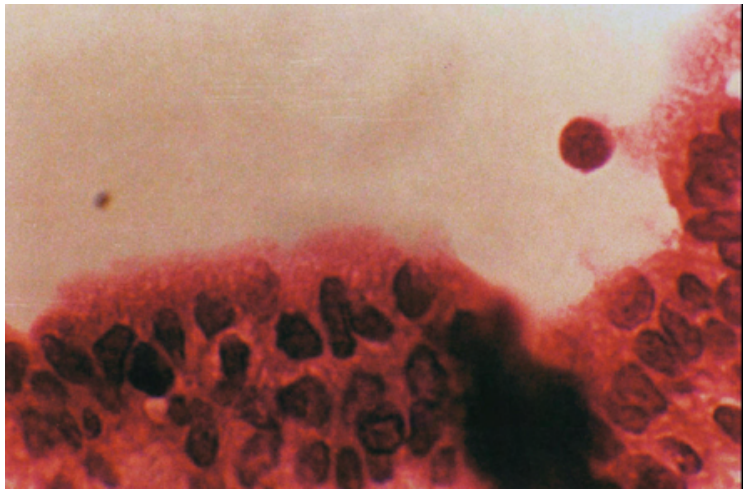

Figure 8: Paroi du kyste avec un revêtement malpighien (coloration Hématéine-Eosine, HE) (Immersion, $\mathrm{x}$ 100)

The cyst wall lined by malpighen epithelium (HE, x 100)

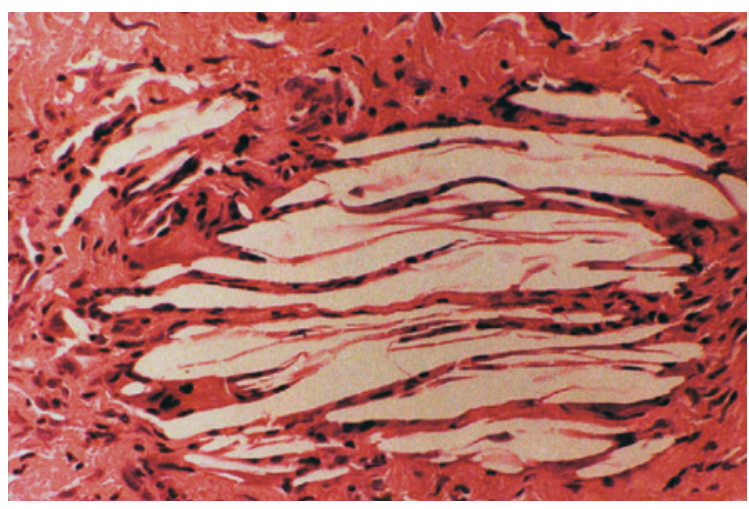

Figure 10 : Réaction granulomateuse autour des cristaux de cholestérol (HE, x 40)

Cholesterol crystals surrounded by granulomatosis reaction (HE, x 40)

ans $[7,8]$. II en est de même pour le cas présenté qui est âgé de 19 ans.

L'étiopathogénie du KNP est complexe et incertaine : il dériverait des vestiges épithéliaux du canal incisif ou naso-palatin. Ce canal est situé à l'extrémité antérieure de la suture médio-palatine qui se forme entre la $8^{\mathrm{e}}$ et la $12^{\mathrm{e}}$ semaine du développement embryonnaire après la fusion des deux processus latéraux palatins du maxillaire $[2,4,5,10]$. En effet, lors du développement embryonnaire, le canal faisant communiquer les cavités buccale et nasale contient en plus des éléments vasculonerveux (artères palatine descendante, sphéno- 
palatine et nerf nasopalatin), des restes du canal nasopalatin embryonnaire qui disparaissent en principe au cours de la $1^{\text {ère }}$ année de la vie. L'involution incomplète des restes de ce canal, d'ailleurs de cause inconnue, donne naissance à des formations kystiques [3, 10].

Plusieurs hypothèses étiopathogéniques ont été évoquées : origine traumatique, infectieuse, glandulaire, génétique, ethnique ou à partir d'organes olfactifs accessoires [3, 5, 6, 8, 10]. Actuellement, certains auteurs pensent qu'il s'agit plus d'une transformation kystique spontanée [4, 6].

Ce kyste se situe exactement sur la ligne médiane entre les incisives centrales supérieures [11]. II est habituellement latent et découvert fortuitement à l'occasion d'un examen radiographique dans 30 à $50 \%$ des cas $[4,6,7,12]$. II peut se révéler par un épisode de surinfection se traduisant par des phénomènes inflammatoires : tuméfaction douloureuse, palatine, rétro-incisive, de consistance ferme, évoluant éventuellement vers la fistulisation, accompagnée le plus souvent par un déplacement des incisives (78,4 \% des cas) $[4,5,6,7,8$, 10, 12, 13]. Parfois, l'extension du kyste se fait en avant donnant une tuméfaction médiane dans le vestibule supérieur [4, 12], comme dans l'observation rapportée. Le diamètre de la tuméfaction dépasse rarement $2 \mathrm{~cm}$. Lorsque le kyste devient volumineux, il peut se développer dans les fosses nasales et dans les cavités sinusiennes [3,11]. Latéralement, il peut s'étendre jusqu'aux prémolaires et ce de façon asymétrique [10]. La vitalité des incisives centrales est en général conservée $[3,4,10]$. L'importance des signes cliniques ne semble pas être en corrélation avec l'âge du patient ou avec la taille du kyste puisque certains kystes relativement volumineux restent asymptomatiques ou sont accompagnés de signes discrets alors que d'autres de petite taille sont accompagnés de manifestations plus importantes $[4,6]$.

Pour l'examen radiographique, les incidences les plus couramment utilisées pour la mise en évidence du kyste sont la radiographie rétroalvéolaire, la radiographie occlusale et l'orthopantomogramme. Ces clichés révèlent un aspect caractéristique de la lésion réalisant une zone radiotransparente bien limitée, ronde, ovoïde ou en cœur de carte à jouer, située entre les incisives centrales supérieures dont les racines sont le plus souvent divergentes [2, 3, 5, 8, 10, 11, 13, 14]. L'aspect caractéristique en cœur de carte à jouer est dû à la projection de l'image de l'épine nasale antérieure ou de la suture palatine médiane sur la lésion kystique radiotransparente [6, 7, 8, 12]. La résorption radiculaire des incisives est exceptionnelle et la lamina dura est toujours conservée $[4,10]$. D'autres moyens d'imagerie comme le scanora, la tomodensitométrie (TDM) et l'imagerie par résonnance magnétique (IRM) se sont révélés très performants pour le diagnostic du KNP $[15,16,17,18]$. Ils permettent une bonne évaluation des limites du kyste dans les trois plans, en particulier l'extension supérieure difficilement appréciable sur les radiographies conventionnelles, et les rapports avec les structures avoisinantes (dents, fosses nasales et cavités sinusiennes) $[15,16]$. Le scanora, qui permet d'adapter les coupes à la courbure du maxillaire, présente aussi l'avantage d'être moins irradiant et moins onéreux que la TDM [17]. La TDM donne un bilan d'extension plus précis : elle permet d'apprécier le degré de résorption de l'os palatin, de préciser s'il persiste une coque osseuse entre le kyste et la muqueuse du plancher des fosses nasales et de donner le rapport exact des apex des incisives centrales avec la cavité kystique, renseignement essentiel pour réaliser le diagnostic différentiel entre KNP et kyste radiculodentaire $[4,15]$. La qualité du contraste tissulaire ainsi que l'absence d'irradiation font de l'IRM un examen de choix pour le diagnostic des kystes en général, et du KNP en particulier [16]. Pour HISATOMI et coll., les signaux recueillis pour le KNP en séquence $\mathrm{T} 1$ et $\mathrm{T} 2$ sont homogènes et d'une intensité élevée, à la différence des autres kystes des maxillaires qui se caractérisent généralement par un signal intermédiaire en T1 [16]. Cette différence d'intensité dans les signaux serait due principalement au contenu kystique relativement visqueux, composé entre autres de vaisseaux et d'une grande quantité de protéines, surtout de la kératine $[16,18]$. Cette constatation présente un double intérêt puisqu'elle permettrait d'orienter le diagnostic avant l'intervention et de choisir la voie d'abord la plus appropriée [16]. médecine

buccale chirurgie buccale

VOL. $11, \mathrm{~N}^{\circ} 1$ 2005

page 35 
L'association kyste fissuraire-dent incluse a été rapportée par Ndiaye-Faty et coll. [1] mais il s'agissait uniquement de kystes fissuraires latéraux. Cliniquement, lorsque le KNP se manifeste par une tuméfaction palatine, le diagnostic différentiel fait évoquer essentiellement une tumeur développée à partir des glandes salivaires accessoires, un fibrome ou un kyste extériorisé du côté palatin $[16,19]$. Radiologiquement, le diagnostic différentiel se fait avec toute lésion se traduisant par une image radiotransparente uniloculaire, à contours nets comme le kyste radiculodentaire généralement appendu à l'apex d'une dent nécrosée, le kyste péricoronaire s'attachant au collet d'une dent incluse [6, 20], le kyste essentiel, l'ameloblastome dans sa forme uniloculaire, le kyste épidermoide, le granulome central à cellules géantes et le fibrome osseux [6, 8, 11, 12, 16, 20]. La présence dans le cas rapporté d'une image médecine buccale chirurgie buccale

VOL. $11, \mathrm{~N}^{\circ} 1$ 2005

page 36 mixte, associant une lacune radiotransparente centrée par une masse radio-opaque, a fait évoquer plusieurs lésions dont le kyste odontogène calcifié. Le diagnostic différentiel radiographique doit aussi prendre en considération l'image radioclaire de la fossette incisive (canal incisif) dont le diamètre ne dépasse pas 6 à10 $\mathrm{mm}$ et dont le contour n'est pas aussi bien délimité que celui d'un kyste [3, 4, 6, 21,22].

Si le diagnostic positif repose sur les données de l'examen clinique et surtout celles de l'examen radiographique, le diagnostic définitf est fourni par l'examen histologique. Ce dernier montre que le KNP est bordé le plus souvent par un épithélium mixte : malpighien dans la portion buccale du canal et cylindrique, pseudostratifié, cilié, de type respiratoire dans sa portion nasale. Parfois, il est bordé par un épithélium uniforme, malpighien ou cylindrique. Des structures épithéliales transitionnelles peuvent être rencontrées, des plages de hyalinisation et des métaplasies sont fréquemment observées dans la paroi du kyste $[2,10]$. La paroi conjonctive est constituée d'un tissu conjonctif dense, riche en vaisseaux et en nerfs auxquels s'adjoignent parfois des glandes muqueuses et du tissu adipeux. Dans la moitié des cas, il existe une réaction inflammatoire comme dans l'observation rapportée [2,10].
Le traitement du KNP est chirurgical, il consiste en l'énucléation totale du kyste [5, 12, 16] suivie de l'examen histopathologique de la pièce opératoire. Les dents, généralement vivantes, doivent être conservées. Le choix de la voie d'abord est en fonction de l'état dentaire, de la taille et de l'extension du kyste dans le sens antéro-postérieur [4]. Dans la plupart des cas, la voie d'abord est palatine en raison de l'évolution palatine plus fréquente du kyste; cette voie minimise le risque de lésion des incisives [4]. Dans le cas rapporté, l'exérèse a été réalisée du côté vestibulaire en raison de l'extension du kyste. La voie d'abord vestibulaire est également choisie chez les patients édentés [4]. L'énucléation doit être complète afin d'éviter toute récidive dont le taux varie de 0 à $11 \%[4,9]$. Le délai de la récidive peut être supérieur à 5 ans d'où la nécessité d'un suivi à long terme [4]. La marsupialisation a proposée dans la littérature surtout pour les kystes volumineux [ 5 , 22]. La régénération osseuse après énucléation se fait lentement ( 3 ans en moyenne) [6, 23], elle est suivie par des examens radiologiques réguliers [3]. Enfin la transformation maligne est exceptionnelle mais reste possible puisque deux cas de carcinomes épidermoïdes intra-osseux développés à partir de l'épithélium non odontogenique d'un KNP ont été rapportés [24,25].

Les kystes épithéliaux non odontogènes sont relativement rares puisqu'ils ne représentent que $5 \%$ des kystes des maxillaires. Le KNP est leur variété la plus fréquente. Le diagnostic différentiel fait évoquer les kystes odontogènes, comme dans le cas présent, ou avec des tumeurs.

Le diagnostic définitif impose la réalisation de l'examen histopathologique de la pièce opératoire même si l'ensemble des signes cliniques et radiologiques est suffisant évocateur du diagnostic.

\section{RÉFÉRENCES}

1 - Ndiaye-Faty Ch, Ba A, Dia Tines S, Niang P, Diallo B, Benslama L. Diagnostic et traitement des kystes épithéliaux non odontogènes : à propos de 14 cas colligés au service de chirurgie buccale du $\mathrm{CHU}$ de Fann. Odonto-stromatologie tropicale 2000 ; 91 : 27-30. 
2 - Le Charpentier Y, Auriol M. Kystes des maxillaires. (pp 7-20). In : Histopathologie bucco-dentaire et maxillofaciale. Y. Le Charpentier, M. Auriol. Ed : Masson, Paris 1997.

3 - Piette E, Reychlen R. Pathologie des maxillaires. (pp 1362-1363). In : Traité de pathologie buccale et maxillo-faciale. E. Piette, R. Reychler. Ed : De Boeck Wesmael, Bruxelles 1991.

4 - Righini CA, Boubagra G, Verougstreate G, Reyt E. Kyste du canal nasopalatin. A propos de 4 cas, revue de littérature. Ann Otolaryngol Chir Cervicofac $2004 ; 121: 115-9$.

5 - Eщiott Kimberly A, Franzese Christine B, Pitman Karen T. Diagnosis and surgical management of nasopalatin duct cysts. Laryngoscope $2004 ; 114$ : 1336-40.

6 - AlbayRam MS, ScIubBa J, ZINREICH SJ. Radiology quiz case: nasopalatine duct cyst. Arch Otolaryngol Head Neck Surg $2001 ; 127: 1283-5$.

7 - ELY N, SCHECHY EC, MC DONALD F. Nasopalatine duct cyst: a case report. Int J Paediatr Dent $2001 ; 11: 135-7$.

8 - VALASQUEZ-SMITH MT, MASON C, COONAR H, BENNETT J. A nasopalatine cyst in an 8-year old child. Int J Pediatr Dent $1999 ; 9: 123-7$.

9 - SWANSOn KS, KaUGRAS GE, Gunsolley JC. Nasopalatine duct cyst: an analysis of 334 cases. J Oral Maxillofac Surg $1991 ; 49: 268-71$.

10 - Favre-Dauvergne E, Auriol M, Le Charpentier Y. Kystes des maxillaires. Encycl Med Chir, 22-062 G 10, 1994.

11 - Horch H. Kystes de la sphère bucco-maxillo-faciale (pp183-238). In : Chirurgie buccale. H. Horch. Ed : Masson, Paris 1996.

12 - BERGEN-SHAPIRO M. Oral pathology quiz 41. Case number 4: nasopalatine duct cyst. J N J Dent Assoc 2003 ; $4: 25-41$.

13 - PHILIPSAPP J, EVERSOLE LR, WYSOCKI JP. Cysts of the oral regions (pp 38-60). In : Contemporary oral and maxillofacial pathology. J PhilipSapp, LR Eversole, JP Wysocki. Ed : Mosby, Saint-Louis 1997.
14 - Cavezian R, PAsquet G. Les images radio-claires extradentaires des maxillaires. (pp 135-140). In : Imagerie et diagnostic en odonto-stomatologie. R Cavezian, G Pasquet. éd :Masson, Paris 1989.

15 - Pevsner PH, Bast WG, Lumerman H, Pivawer G. CT analysis of a complicated nasopalatine duct cyst. N Y State Dent J 2000 ; 66 : 18-20.

16 - HISATOMI M, ASAUMI JI, KONOUCH H, MatSUZAKI H, KISHI K. MR imaging of nasopalatine duct cysts. Europ J Radiol $2001 ; 39$ : 73-6.

17 - HaRRIS IR, BRown JE. Application of cross-sectional imaging in the differential diagnosis of apical radiolucency. Int Endo J 1997; 30 : 288-90.

18 - Robertson H, Palacios E. Nasopalatine duct cyst. Ear Nose Throat J 2004 ; 83 : 313.

19 - Wood NK, DoAz PW. Periapical radiolucencies. (pp 252277). In : Differential diagnosis of oral lesions. NK. Wood, PW. Doaz. Ed : Mosby, Saint-Louis 1991.

20 - Guilbert F, Chomette G, Le Charpentiery, Auriol M. Les tumeurs bénignes et les pseudo-tumeurs des maxillaires. Rev Stomatol Chir Maxillofac 1993 ; 94 :197-265.

21 - CAWson RA, BInNie WH, EVESON JW. Cysts and cystlike lesions of the jaw (pp $5: 13-5: 14$ ). In : Color atlas of oral disase: clinical and pathologic correlations. RA.Cawson, WH. Binnie, JW.Eveson. Ed : Wolfe, USA 1994.

22 - Vasconcelos RF, Ferreira de Aguiar MC, Castro WH, Cavalcanti de Araujo V, Mesquita RA. Retrospective analysis of 31 cases of nasopalatine duct cyst.Oral Dis $1999 ; 5: 325-8$.

23 - AnNeROth G, Hall G, Stuge U. Nasopalatine duct cyst. Int J Oral Maxillofac Surg $1986 ; 15$ : 572-8.

24 - TAKAGI R, OHASHI Y, SUSIKI M. Squamous cell carcinoma in the maxilla probably originating from a nasopalatine duct cyst: report of case. J Oral Maxillofac Surg 1996 ; $54: 112-5$.

25 - TAKEDA Y. Inta-Osseous squamous cell carcinoma of the maxilla: Probably arisen from non-odontogenic epithlium. Br J Oral Maxillofac Surg1991; 29 : 392

\begin{tabular}{l}
$\begin{array}{l}\text { médecine } \\
\text { buccale } \\
\text { chirurgie } \\
\text { buccale }\end{array}$ \\
\hline VOL. 11, N ${ }^{\circ} 1$ \\
2005 \\
\hline page 37
\end{tabular}

page 37 